\title{
L'ethos discursif
}

\section{Maria da Glória Corrêa di Fanti ${ }^{1}$ \\ Liz Feréz}

1 Pontifícia Universidade Católica do Rio Grande do Sul, Porto Alegre, Rio Grande do Sul, Brasil. 2 Université Paris VIII, Centre d'Études sur les Médias, les Technologies et I'Internalisation, Paris, France.

$\mathrm{L}$ a notion d'ethos discursif est devenue un objet de réflexion pour différentes recherches qui étudient l'image de l'énonciateur produite dans le discours. Selon Maingueneau (2005, 2006, 2008, 2014), cette image se construit dans les discours par le biais de ses multiples relations avec l'autre (sujets et discours) et émerge de l'articulation entre divers éléments (verbales et non verbales, éthiques et esthétiques etc.), qui requièrent l'incorporation de l'interlocuteur pour l'appréhender dans un ensemble complexe de représentations sociales et culturelles ${ }^{1}$.

L'ethos discursif, bien qu'il diffère de la tradition rhétorique, respecte trois idées fondamentales de l'ethos aristotélicien : il est $a$ ) une notion discursive, c'est-à-dire qu'il se constitue à travers le discours et n'est donc pas une "image du locuteur extérieure à la parole", b) un "processus interactif d'influence sur l'autre" et c) une notion "hybride (socio-discursive), ayant un comportement socialement évalué, qui ne devrait pas être saisi hors d'une situation de communication précise" et qui est propre à un contexte historico-social (MAINGUENEAU, 2006, p. 60).

Dans une perspective discursive, tout texte présente une vocalité qui peut être corrélée à la "caractérisation du corps de l'énonciateur" construite dans le discours (MAINGUENEAU, 2006, p.61). L'ethos couvre non seulement la dimension verbale, mais également un ensemble de caractéristiques psychiques et physiques qui s'associent à un garant, lequel se révèle à travers un ton attestant ce qu'il dit. Le garant reçoit ainsi un caractère et une corporalité qui varieront en fonction de la constitution des textes, de la scène de la parole créée. Alors que "le caractère correspond à un faisceau de traits psychologiques", la corporalité est associée à une

\footnotetext{
1 Une partie des idées sur l'ethos a été développée dans l'article Identidade, alteridade e cultura regional: a construção do ethos milongueiro gaúcho (DI FANTI, 2009).
}

"complexion physique et à une manière de s'habiller" (MAINGUENEAU, 2006, p. 62).

Outre le caractère et la corporalité, l'ethos "implique une manière de se mouvoir dans l'espace social, une discipline tacite du corps appréhendé à travers un comportement" (MAINGUENEAU, 2006, p. 62). Dans cette perspective, l'incorporation de l'interlocuteur ne s'effectue pas par la simple identification d'un caractère garant, mais par l'implication dans "un monde éthique", qui, selon l'analyste du discours, fonctionne comme un ensemble diffus de représentations sociales et culturelles, ce qui rend manifeste une tension entre l'ethos désiré et l'ethos effectivement élaboré.

Amossy, en abordant la question de l'ethos et de l'identité verbale, observe que le locuteur produit, dans le discours, une diversité d'images de lui-même, qui révèle des facettes de son identité. Ces caractéristiques identitaires peuvent aussi projeter des représentations de groupes, dans lesquelles l'individu apparaît comme "le représentant d'une collectivité et où le discours peut apparaître comme issu de plusieurs voix confondues" (AMOSSY, 2010, p.211). Ainsi, la présentation de soi dans le discours peut renvoyer non seulement à des groupes aux contours encore vagues, mais aussi à des groupes reconnus dans l'espace social, qui souhaitent, par exemple, une reconnaissance identitaire ou s'affirmer par le biais de relations de pouvoir.

La présentation de soi dans le discours, qui confère une autorité au locuteur, permet, d'un côté, à des degrés divers, l'émergence d'affinités et de rapprochements avec certains individus, et, de l'autre, l'émergence de distinctions et de distanciations. Selon Amossy, comprendre l'image, qui est construite dans le discours, individuel ou collectif, implique des réflexions sur l'ethos préalable et l'ethos discursif. Alors que le premier présuppose la connaissance du mode d'être du locuteur, le second nécessite l'observation du discours à travers lequel 
le locuteur projette une image de lui-même et négocie son identité (AMOSSY, 2010, p.212). D'après l'auteure, il n'existe aucun moyen de dissocier une instance de l'autre, car le locuteur dans le discours se réfère à des données antérieures, en les resignifiant. Dans la négociation de la construction de l'image de soi, le locuteur dialogue avec ce que les autres disent et pensent de sa personne.

Le présent dossier, qui ouvre la voie au débat sur la portée, les défis et les potentialités de la notion d'ethos discursif, regroupe, dans cette édition de Letras de Hoje, un ensemble d'articles centrés sur la production, la circulation et la réception de différents discours dans la société. L'article de Dominique Maingueneau, qui ouvre ce numéro, développe une réflexion critique sur la notion d'ethos discursif, à partir de sa contribution, depuis les années 1980, au développement de cette problématique. Sa réflexion prend en compte la diversité des manifestations du discours, le processus de construction de l'ethos et la relation étroite entre les dimensions verbale et iconique. Ses considérations se fondent sur l'analyse des sites internet et des publicités. L'article suivant, de Maria Cecília Pérez Souza-e-Silva et Décio Rocha, discute l'interrelation entre la scénographie et l'ethos de Maingueneau.
L'analyse des discours circulant sur internet et/ou les réseaux sociaux est menée par un bon nombre de chercheurs, qui articulent des réflexions théoriques et pratiques, tels Juan Manuel López Muñoz et Tamiris Machado Gonçalves, Cristina Rothier Duarte et Francisco Vieira da Silva, Bianca Assis Oliveira de Paula, Fabio Sampaio de Almeida et Maria Cristina Giorgi, Eliane Davila dos Santos et Gislene Feiten Haubrich, Ernani Cesar de Freitas et Luis Henrique Boaventura.

L'analyse des discours véhiculés par des revues est développée par Luciana Salazar Salgado et Marina Delege, Elaine Cristina Fonseca, Luana Ferreira de Souza. Dans le champ culturel, Hélène Barthelmebs-Raguin, Lia Raquel Vieira de Andrade et Juliana Georgia Gonçalves Araújo nous présentent leurs réflexions. Dans le domaine de l'enregistrement téléphonique, Welton Pereira e Silva travaille sur la question de l'arnaque du faux enlèvement. Enfin, la publicité est traitée par Silma Ramos Coimbra Mendes et Edgar Godoi Gabriel.

Nous sommes reconnaissants aux auteurs du présent numéro pour leur importante collaboration et invitons la communauté universitaire à découvrir leurs réflexions.

\section{Références}

AMOSSY, R. (Org.). La présentation de soi. Ethos et identité verbale. Paris: PUF, 2010.

DI FANTI, M. G. C. Identidade, alteridade e cultura regional: a construção do ethos milongueiro gaúcho. Alfa: Revista de Linguística (UNESP. Online), v. 53, p. 149-166, 2009.

MAINGUENEAU, D. Retour critique sur l'éthos. Langage et société, v. 149, n. 3, p. 31-48, 2014.

MAINGUENEAU, D. A propósito do ethos. Tradução de Luciana Salgado. In: MOTTA, A. R.; SALGADO, L. (Org.). Ethos discursivo. São Paulo: Contexto, 2008.

MAINGUENEAU, D. Cenas da enunciação. Organizado por Sírio Possenti e Maria Cecília Pérez de Souzae-Silva e tradução de Sírio Possenti. Curitiba: Criar, 2006.

MAINGUENEAU, D. Ethos, cenografia, incorporação. In: AMOSSY, R. (Org.). Imagens de si no discurso: a construção do Ethos. Tradução de Dílson Ferreira da Cruz, Fabiano Comesu e Sírio Possenti. São Paulo: Contexto, 2005.

\section{Organisatrices}

(D) MARIA DA GLÓRIA CORRÊA DI FANTI <gloria.difanti@pucrs.br>

Professeure-chercheuse à la PUCRS et boursière du CNPq.

(D) LIZ FERÉ <info@lizfere.com>

Professeure-chercheuse à l'Université Paris VIII/CEMTI. 